\title{
Opportunistic Scheduling with Reduced Feedback
}

\author{
Husni I. H. Abu Arja and Mehrdad Dianati \\ Centre for Communications Systems Research (CCSR) \\ Department of Electronic Engineering \\ University of Surrey \\ Guildford, GU2 7XH, United Kingdom
}

\begin{abstract}
- this paper investigates and proposes the techniques that reduce the number of required feedback channels for implementation of opportunistic scheduling. It is shown that by implementing effective schemes we can exploit significant multiuser diversity gain using a modest number of feedback channels. Simulation based performance analysis is given to demonstrate effectiveness of the proposed schemes in terms of high spectrum efficiency and low feedback overhead.
\end{abstract}

Index Terms - Feedback channels with different threshold range, Opportunistic scheduling with maximum channel utilization.

\section{INTRODUCTION}

SCHEDULING of non-real-time data transmission to users is an effective technique for bandwidth sharing in communication networks [1][2] for most of data applications such as web traffic. In particular, opportunistic allocation of access to a shared and slowly fading wireless channel to the users with a better quality of channel in each scheduling epoch results in a higher spectral efficiency as the number of users increases. This gain in channel utilisation is known as the multi-user diversity gain [3] in wireless communication systems. Implementation of opportunistic scheduling requires knowledge of partial Channel State Information (CSI) in each and every scheduling epoch. Given that channel variations due to the fading process are slow, the CSIs in the current timeslot (scheduling epoch) could be used to make a reasonable estimation channel states in the upcoming timeslots. Pure opportunistic scheduler could result in unfair allocation of channel resources among the users who enjoy different average quality of channel. Thus, fairer amendments of opportunistic scheduling, e.g., Proportional Fair Scheduling [4] was introduced later in order to address this problem.

Although opportunistic scheduling is an optimal resource allocation scheme in terms of spectrum utilisation [3], the amount feedback information for implementation of this scheme could be immense. Thus, reduction of feedback channels for opportunistic scheduling becomes an important problem that has been investigated by researchers in the recent years. These studies use a variety of techniques to tackle the problem. A threshold based algorithm has been proposed in [4], where each user compares its current CSI with a threshold level, which will is selected and circulated by the base station, in each timeslot. In this solution, those users with lower CSI than the threshold level will not transmit their channel status to the base station. This scheme helps reduce the amount of feedback information; however, the cost is lower multiuser diversity gain than the classical opportunistic scheduling. The trade-off between multiuser diversity gain and feedback information reduction can be made by adjusting the level of the threshold in this scheme. Alternatively, an Automatic Repeat request (ARQ) based scheme for feedback information reduction has been proposed in [5]. This scheme exploits the existing ARQ signalling in the link layer to piggyback occasional feedback information from the users that is used for CSI estimation in conjugate with a Finite State Markov channel Model (FSMC). This scheme does not need any explicit feedback channels as it relies on the existing ARQ signals. However, error due to channel estimation could lead to reduction of the spectrum efficiency.

Feedback reduction for proportional fair opportunistic scheduling has been studied in [6]. This study proposes a scheme similar to that of [4], but the threshold in this work is related to the normalized SNR of the users. This scheme provides a strict fairness with a reduction in the number of feedback channels and the amount of feedback information. However, the number of feedback channels in this scheme increases as the number of users increase. In [7] and [8] a threshold-based scheme, with a fixed number of mini slots for feedback information that precede the transmission slot, has been proposed. In [7] a random access protocol is used, while in [8] a contention based scheme with maximum quantile scheduling scheme is utilised. The results given in [7] and [8] indicate reduction in feedback information; however, these schemes obviously cannot proportionally enjoy multiuser diversity gain as the number of users increases. In [9], multiple feedback thresholds are proposed in order to reduce the number of users transmitting their CSI by polling the users with the highest threshold first, and the lower ones, later. This scheme reduces the number of users transmitting their CSI, while maintaining high spectrum efficiency. For a large number of users, this scheme can significantly reduce the number of users that need to feedback their CSI to the base station by increasing the number of thresholds. The drawback of this scheme however is the time that is needed in order to poll all the users over the number of thresholds.

This paper proposes hybrid schemes that adopts some aspects of the previous solutions in order to overcome their weaknesses in terms of reducing feedback while maintaining a close spectrum efficiency to that of a the classical opportunistic scheduling. The first proposed scheme, called Scheme (a), uses adaptive threshold levels, depending on the real-time traffic load. In this scheme, we consider the real- 
time traffic load to be independent of non-real-time traffic. Real-time traffic is the highest priority traffic that is served without scheduling. The remaining resources are utilised by the scheduler to serve non-real-time traffic. In Scheme (a), if the real-time traffic load is low, a smaller threshold level will be chosen to boost the multiuser diversity gain of the opportunistic scheduler. In contrast, when the real-time traffic load is high, Scheme (a) will choose a larger threshold level to reduce the number of required feedback channels. The second proposed scheme, called Scheme (b), uses a single threshold level and limits the number of feedback channels. In this scheme the eligible users who enjoy better than threshold quality of channel, will compete for one of the available feedback channels. Then, we propose Scheme (c) that aims to choose an optimum value for the threshold level and the number of feedback channels in order to minimize feedback overhead and maximize multiuser diversity gain. Finally, in order to demonstrate the efficacy of the proposed schemes and compare their performance Monte Carlo simulations are performed and the results are presented.

The rest of this paper is organised as follows. In Section II, the system model is introduced. The proposed schemes are discussed in Section III. The results of the performance analysis are given and discussed in Section IV. Finally, the concluding remarks are made in Section V.

\section{SySTEM MODEL}

The system model that is considered in this paper consists of a single base station with a single antenna and $N$ mobile users each equipped with a single antenna, as shown in Figure 1. The channels will be considered as Rayleigh channels and an assumption of no errors or delay will be associated with the feedback channels. Scheduling is implemented for the nonreal-time data traffic. Downlink scheduling is the primary focus of this work as spectral efficiency becomes more crucial for the downlink due to the asymmetric nature traffic in data applications. A saturated scenario is considered, where there is always backlogged data for transmission to every user. Scheduling decisions are be made by the base station at the beginning of each timeslot. Duration of each timeslot is $1.25 \mathrm{~ms}$. Fading process is considered to be slow such that the quality of channel to a user remains constant for the duration of at least 3 timeslots. Thus, the base station could reliably use the feedback information from the users.

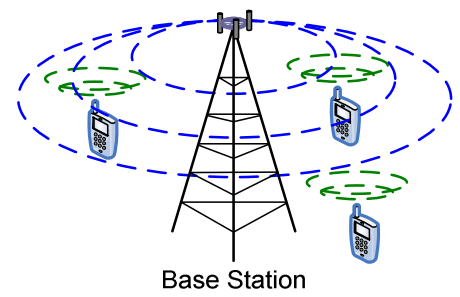

Figure 1. System model scenario

The base station will select one of the $N$ simultaneously active users with the best channel status for each timeslot with a data-rate of $r_{k}$ that depends on the quality of channel in terms of Signal to Noise and Interference Ration (SINR). The mapping between SINR values and the achievable rates depends on the characteristics of the physical layer which is usually arranged in a lookup table with a finite number of rows. The table is defined by dividing the SINR range into $m$ intervals by defining $(m-1)$ different SINR levels, $\left\{\zeta_{1}, \zeta_{2}, \ldots, \zeta_{m-1}\right\}$, where $\zeta_{1}<\zeta_{2}<\ldots<\zeta_{m-1}$. Then, the corresponding achievable rates can be represented by the set $\mathfrak{R}=\left\{r_{1}, r_{2}, r_{3}, \ldots, r_{m}\right\}$, where $r_{i}$ corresponds to the SINR $\operatorname{interval}\left[\zeta_{i-1}, \zeta_{i}\right]$. Each rate is achieved by selecting a certain Modulation and Coding Scheme (MCS) defined by the physical layer.

\section{OPPORTUNISTIC SCHEDULING AlgORITHMS}

In this section, the details of the proposed schemes are discussed.

\section{a. Scheme (a): Opportunistic Scheduling with Threshold Adaptation}

The idea behind this scheme is to allow more users to feedback their CSI if the base station can afford more feedback channels and vice versa. This is implemented by adapting the active threshold level according to the available system resources for non-real time traffic. This is determined by the load of real time traffic which has higher priority. When the demand for real-time traffic is high, the base station prefers to allocate fewer resources for feedback channels; thus, it applies a policy that reduces the number of users that report their CSI in each timeslot. The policy is implemented as follows. The base station broadcasts a set of predefined threshold levels denoted by $\left\{\gamma_{1}, \gamma_{2}, \gamma_{3}, \ldots, \gamma_{x}\right\}$, where $x$ is the total number of thresholds levels. Then, the base station periodically selects and broadcasts the index of the active threshold level, $\gamma_{t h r}$, which is dictated by real-time traffic load in the system, to be used by the mobile users. In this scheme, a mobile user will compare the quality of its channel, $\gamma_{i}$, with the active threshold value, $\gamma_{t h r}$. If $\gamma_{i} \geq \gamma_{\text {tht }}$, then, the user will feedback its CSI to the base station. Having collected the CSIs, the base station will select the best user and the appropriate MCS for the selected user.

This scheme can achieve a good performance in terms of spectrum utilisation, as will be demonstrated by the performance analysis results later in this paper. However, the random limit imposed by the level of real-time traffic, may constraint the achievable multiuser diversity gain as the number of user increases. This is due to the fact that multiuser diversity gain will not only depend on the number of non-real-time users, served by the scheduler, it will also depend on the number of real-time users, that are not served by the scheduler. Increasing load of non-real-time traffic will have an adverse effect on the achieved multiuser diversity gain by the scheduler. 


\section{b. Scheme (b): Opportunistic Scheduling with Threshold and Limited Number of Feedback Channels}

In the previous scheme the number of required feedback channels increases as the number of users increases. This may not be desirable in some systems. Thus, the second proposed scheme imposes a hard limit on the number of feedback channels, denoted by $M$. Similar to the previous scheme a threshold level is used to reduce the competition for the feedback channels by deferring the users with poor channel condition in a given timeslot.

In this scheme, each user will measure its channel quality, and compare it with the predefined threshold level. If the channel quality of a user exceeds the threshold level, it will contend for a feedback channel. The contention among qualified mobile stations is resolved by a random access mechanism. If the number of qualified users is more than $M$, the random access process will randomly select $M$ lucky users who will get to report their CIS back to the base station. Otherwise, all of the qualified users will have the opportunity to report their CSI to the base station.

This scheme does not have the drawback of increasing number of required feedback channels. However, a hard limit on the number of feedback channels will obviously have a modest adverse effect on the achievable multiuser diversity gain when the number of number of users in the system is moderate. In addition, the contention mechanism will increase the access delay.

\section{c. Scheme (c): Opportunistic Scheduling with Limited Number of Feedback Channels and Adaptive Threshold}

The performance study of the proposed (b) indicates that the multiuser diversity gain of an opportunistic scheduler with a limited number of feedback channels can be optimised by choosing a proper threshold value for any given number of users with non-real-time traffic. This property is illustrated by the examples in Figure 2. This figure indicates that there is an optimum threshold level that maximises the total system throughput for any given number of users with non-real-time traffic. In this example the number of available feedback channels is five.

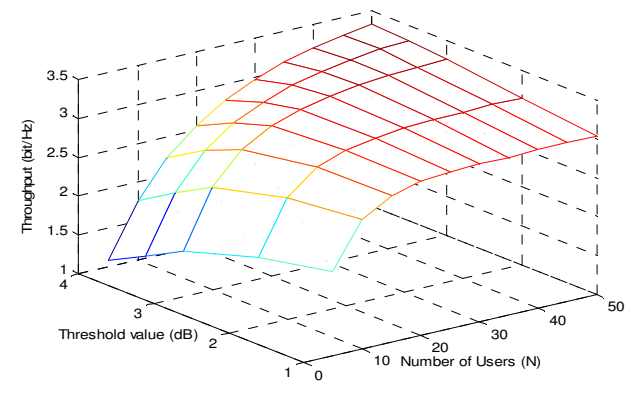

Figure 2. The total throughput of Scheme (b) for different number of non-real-time users and threshold values (5 feedback channels).

Although the exact value of the threshold level will depend on other system parameters such as the average channel gains and etc, this knowledge can be exploited by the base station.
For instance a base station can develop lookup tables in order to assist selecting efficient threshold values.

\section{d. Scheme (d): Opportunistic Scheduling with Limited Feedback Channels and Adaptive Multiple Threshold}

Scheme (d) aims to use the implicit knowledge about the status of user channels to reduce the number of required feedback channels to only three channels. This is accomplished by intelligently selecting the active threshold levels, and using the feedback channels to allow a limited number of users with good channel conditions to compete for reporting their CSIs back to the base station.

In this scheme, the range of the receiver side Signal to Noise and Interference Ratio (SINR) is divided into several intervals as shown in Figure 3. In this model, the channel for a mobile user is considered to be in state $\mathrm{Si}$, when the received SINR for that user is in interval $\left[\gamma_{i-1}, \gamma_{i}\right)$. As it is indicated in this figure, for the terrestrial mobile communication systems the probability of transition among non-adjacent states is negligible for the reasonably close time instants. Each state usually corresponds to a specific Modulation and Coding Scheme (MCS) in the physical layer, resulting in a certain transmission rate.

At the beginning, Scheme (d) assigns the three feedback channels to three randomly chosen and adjacent states. Then, the users are instructed to compete for CSI reporting using a specific feedback channel if their SINR level is in the range associated with that feedback channel. If the SINR level of a user does not match that of any feedback channel, it will defer from CSI reporting. If the base station receives CSI reports in all three feedback channels, this indicates that there are candidate users in all three SINR levels associated with the three feedback channels. At this stage, the base station cannot make sure that there are no candidate users with better channel qualities in the higher but unexplored states.

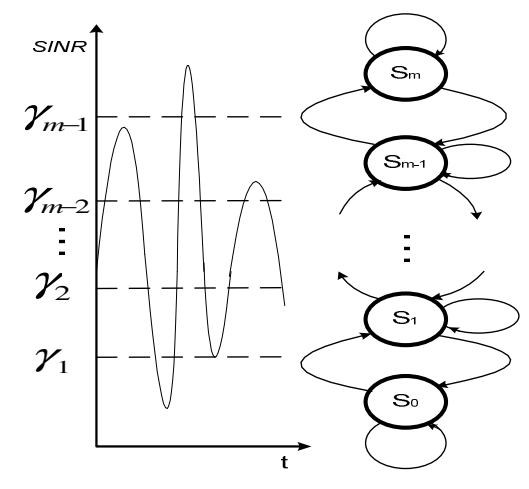

Figure 3. Definition of multiple channel states.

Thus, the base station associates the three available feedback channels with next three higher and unexplored states in Figure 3. This process may need to be repeated one or two more times to the best user is found. If in the first trail there is no qualified user in any of the three feedback channels, the base station will examine three unexplored lower states in the next trail. In steady state, the base station will develop a better knowledge from which states it needs to start polling 
process to minimize the number of trails. In practice, the base station will rarely need to perform polling more than once.

The amount of feedback information in Scheme (d) is very low. Indeed, an eligible user will send only one bit of information on the feedback channel to indicate that its SINR value qualifies for the MCS associated with a certain feedback channel in a polling round.

\section{Simulation ReSults}

In this section, simulation based performance analysis of the proposed schemes in this paper is given. A system model consisting of a single base station and multiple mobile stations is considered. The results could be applicable for a multi-cell scenario if the interference from multiple surrounding cells is Gaussian. The simulation system operates at $f_{c}=1900$. Rayleigh fading channels are simulated by filtering of two independent white Gaussian processes. The scheduling decisions are made every timeslot; the duration of each timeslot is $1.25 \mathrm{~ms}$.

Figure 4 compares the total throughput of Scheme (a) with that of the classical Opportunistic Scheduling (OS). The graph shows that classical OS outperforms scheme (a), particularly, when the number of non-real-time users is relatively small. The decline in the spectrum efficiency in scheme (a), when the number of non-real-time users is relatively small, is due to the difficulty of adjusting the threshold level. Thus, there is a chance that in some timeslots there will be no qualified users. However, as the number of users increases, this even will be less likely.

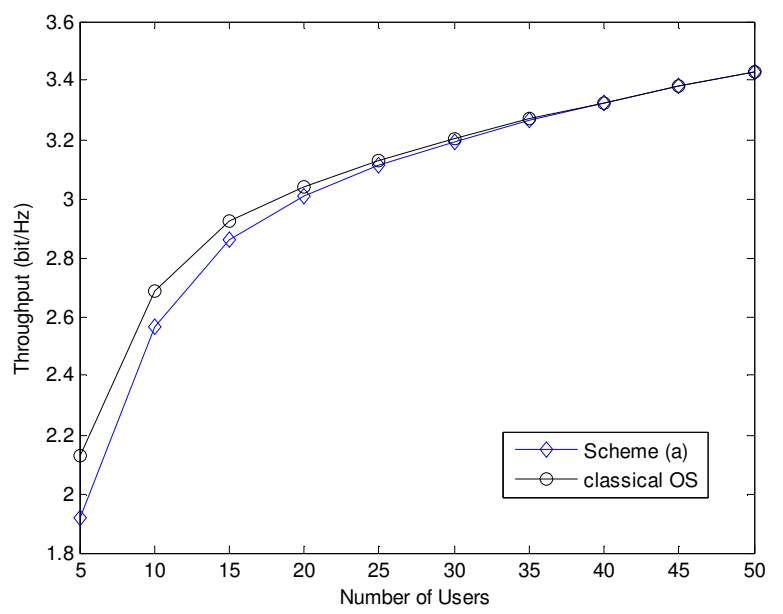

Figure. 4. Scheme (a): Comparison of the total throughput of Scheme (a) with that of the classical Opportunistic Scheduling (OS)

Figure 5 compares the feedback overhead, in terms of the number of feedback channels, of scheme (a) and classical OS. This figure demonstrates significant reduction in the number of feedback channels using Scheme (a) instead of classical OS. However, as it can be seen, still the number of feedback channel for the both scheme increases with the number of non-real-time users.
Figure 6, shows the spectrum efficiency of scheme (b) at different threshold values. This figure shows that smaller threshold levels can achieve higher spectrum efficiency. However, higher thresholds are preferred when the number of non-real-time users is higher.

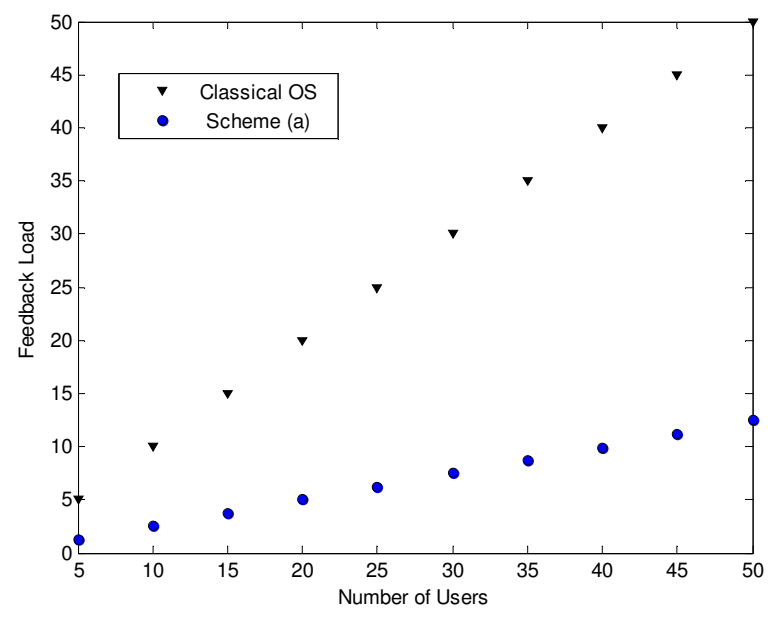

Figure. 5. Scheme (a): Comparison of the feedback overhead, in terms of the number of feedback channesl, for Scheme (a) and classical OS.

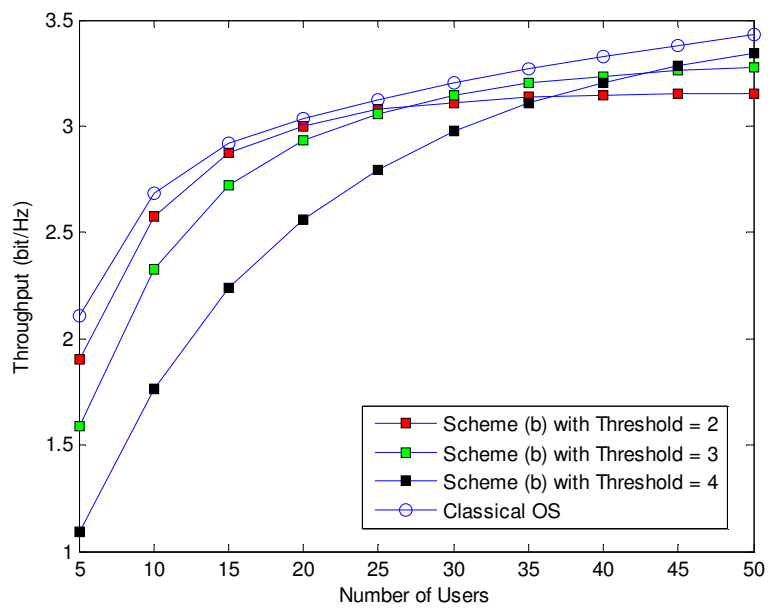

Figure. 6. Performance of Scheme (b) for different thresholds levels

The number of feedback channels for different threshold levels in Scheme (b) is shown in Figure 7. This figure shows that the numbers of feedback channels increases with the increasing number of non-real-time users in the system. However, unlike Scheme (a), the number of feedback channels is limited to $M$ feedback channels.

Figure 8 compares spectrum efficiency of Scheme (b) with that of a classical OS. This figure shows that by adapting the threshold value according to the number of non-real-time users we can improve spectrum efficiency using only a limited number of feedback channels. Scheme (c) obviously outperforms Scheme (b); however, it suffers from the complexity of finding the appropriate threshold for the different group of users. 


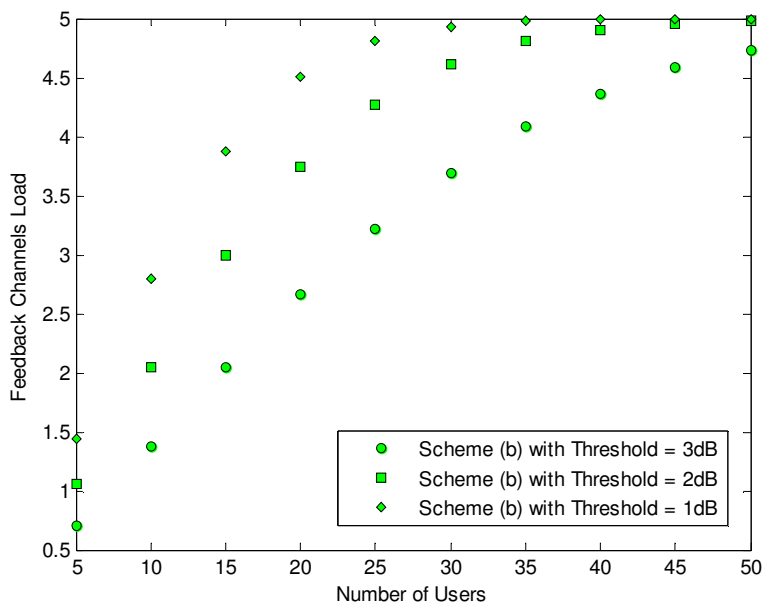

Figure. 7. The number of required feedback channels in Scheme (b)

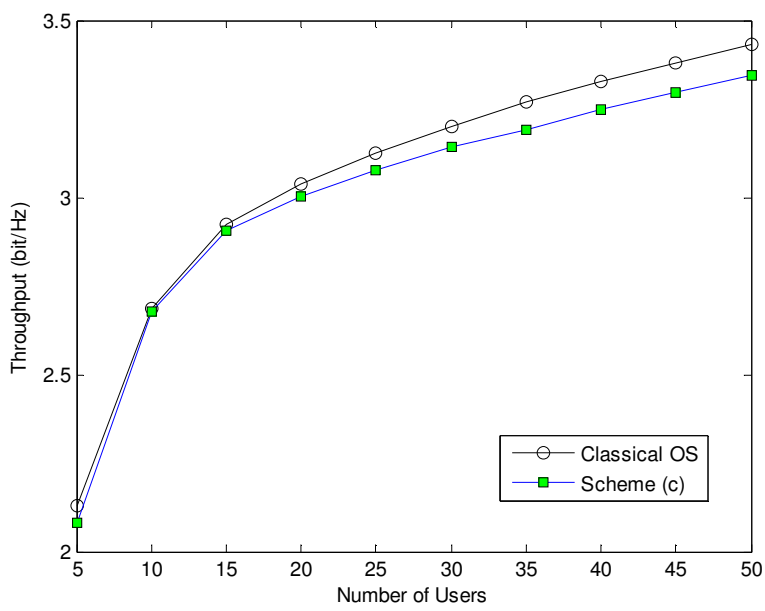

Figure. 8. Comparison of the performance of Scheme (c) with that of classical OS

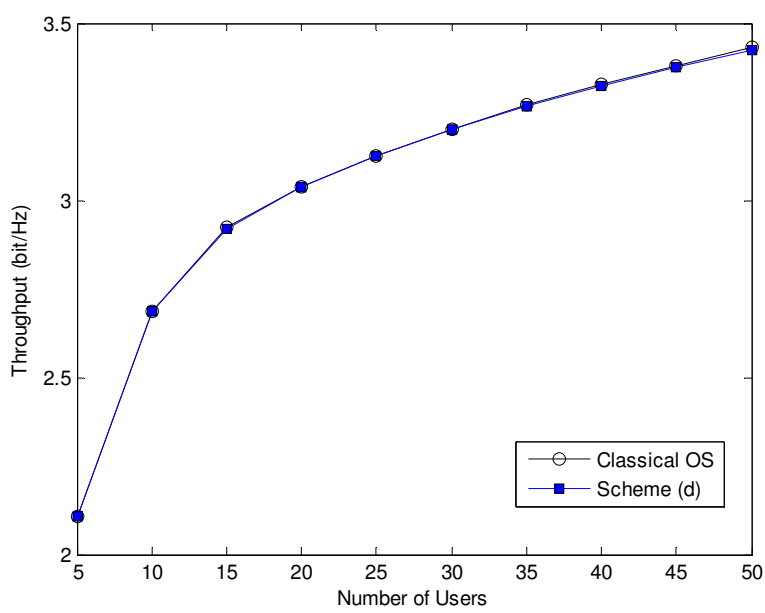

Figure 9. Comparison of the spectrum efficiency of Scheme (d) with that of a classical OS
Figure 9, shows that the performance Scheme (d), that uses only three feedback channels, is very close to that of a classical OS, where a dedicated feedback channel is needed for each non-real-time user. In comparison to Scheme (c), this scheme does not need any complex mechanisms for threshold level adaptation. However, the drawback of Scheme (d) is the latency introduced the polling process. In addition, in fast fading environments, Scheme (d) may be less effective due to channel estimation errors.

\section{Conclusions}

In this paper, we investigated a variety of techniques that can be used to reduce feedback overhead for implementation of opportunistic resource allocation in wireless networks. It is shown that by using threshold based scheme and adapting the threshold levels we can harvest multiuser diversity gain while reducing the number of required feedback channels. In addition, it is shown that smart polling mechanisms can help use only limited number of feedback channels. For example, by using only three feedback channels and an effective polling mechanism, we can achieve a spectrum efficiency level that is very close to that of a classical opportunistic scheduler.

\section{ACKNOWLEDGEMENT}

This work is supported by the Engineering and Physical Science Research Council (UK) of the United Kingdom under India UK Advanced Technology Centre (IU-ATC) grant.

\section{REFERENCES}

[1] H. Zhang, "Service Disciplines for Guaranteed Performance Service in Packet-switching Networks," in Proc. IEEE, vol. 83, Oct.1995, pp.1374-96.

[2] X. Liu, E. Chong, N. Shroff, "Transmission Scheduling for Efficien Wireless Utilization," in Proc. INFOCOM 2001, Anchorage, v. 2, pp. 776-785.

[3] D.N.C Tse, "Optimal Power Allocation over Parallel Gaussian Channels," in Proc. Of Int. Symp. Info. Theory, June 1997, pp. 27.

[4] A. Jalali, R. Padovani, R. Pankaj, "Data throughput of cdma HDR: a high efficiency-high data rate personal communication wireless system," in Proc. IEEE VTC, May 2000.

[5] D. Gesbert, and M. Slim-Alouini, "How Much Feedback in Multi-User Diversity Really Worth?," in Proc. IEEE International Conf. on Comm., 2004, June 2004, pp. 234-238.

[6] M. Dianati, R. Tafazolli, "Opportunistic Scheduling over Wireless Fading Channels without Explicit Feedback," in Proc. IEEE VTC Spring 2008, pp. 1871-1875.

[7] G. U. Hwang, F. Ishizaki, "Design of a Fair Scheduler Exploiting Multiuser Diversity with Feedback Information Reduction," in Proc. IEEE Comm. Letters, 2008, February 2008, vol. 12, pp. 124-126.

[8] T. Tang, R.Heath, "Opportunistic Feedback for Downlink Multiuser Diversity," IEEE Comm., Letters., vol. 9, pp. 948-950, Oct. 2005.

[9] S. Patil, G. de Veciana, "Reducing Feedback for Opportunistic Scheduling in Wireless Systems," IEEE Trans. on Wireless Comm., vol. 6, pp. 4227-4232, December 2007.

[10] V. Hassel, D. Gesbert, M.-S. Alouini, G.E. Oien,“A Threshold-Based Channel State Feedback Algorithm for Modern Cellular Systems," IEEE Trans. on Wireless Comm., vol. 6, pp. 2422-2426, July 2007. 